\title{
El Tecnólogo Médico en Terapia Física y Rehabilitación en tiempos de pandemia Covid 19 en Perú
}

\section{The Medical Technologist in Physical Therapy and Rehabilitation at Covid 19 Pandemic Times in Peru}

Cristhian Santiago-Bazán 1,2,a,b

\section{Señor editor,}

En la actualidad se vive una coyuntura preocupante a nivel internacional y nacional. A la llegada al Perú desde el primer caso el 15 de marzo se estableció el Estado de Emergencia Nacional por las graves circunstancias que afectan la vida de la Nación a consecuencia del COVID19 , conllevando a un aislamiento social obligatorio. Teniendo como grupos de mayor riesgo laboral en esta pandemia al personal de salud ${ }^{(1)}$, entre ellos tenemos a los profesionales tecnólogos médicos de las diferentes áreas de la carrera profesional de Tecnología Médica: laboratorio clínico, radiología, terapia física y rehabilitación, Terapia de lenguaje, terapia ocupacional, optométria.

El decano del Colegio de Tecnólogo Médico del Perú, reafirma su compromiso en la lucha contra el coranovirus, haciendo mención que el profesional tecnólogo médico representa el $70 \%$ de profesionales de la salud se encuentran en la lucha contra el coronavirus Covid-19) el cual solicita solidez y sostenibilidad política en el sector salud ${ }^{(2)}$.

Así mismo, entre las competencias del tecnólogo Médico en Terapia Física y Rehabilitación en el Perú tenemos: es un profesional independiente, altamente capacitado, con espíritu crítico, que actúa directamente con las personas, realizando la evaluación, diagnóstico físico - funcional, programación y tratamiento para la promoción y prevención, mantenimiento $\mathrm{y} / \mathrm{o}$ restablecimiento funcional en problemas de salud ${ }^{(3)}$, el cual pertenece a la carrera de Tecnología Médica amparado bajo la Ley 28456.

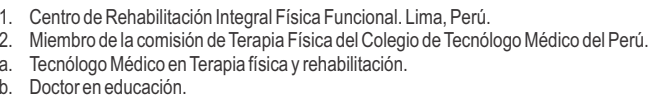

La pandemia por Covid-19 está modificando el perfil de discapacidad por varias razones: el aumento de déficits secundarios propios de la enfermedad, el incremento de déficit funcionales en pacientes que han dejado de atenderse y el impacto del descenso en la actividad física por el confinamiento, principalmente en las personas de la tercera edad, frágiles o con discapacidades, el cual es un nuevo reto y una oportunidad $^{(4)}$.

La participación del especialista en terapia física respiratoria, se propende por realizar intervenciones, para minimizar los efectos funcionales en dichos pacientes con Covid-19, juega un papel primordial, no solo en la fase aguda de la enfermedad por Covid-19 en las unidades de cuidados intensivos y en los pacientes hospitalizados. Así mismo es la piedra angular en el equipo interdisciplinar de salud para la recuperación de las secuelas que esta enfermedad pueda dejar a nivel de función pulmonar y de capacidad funciona $l^{(5)}$.

Los efectos secundarios a consecuencia del reposo prolongado, las secuelas sobre el sistema respiratorio y otros sistemas, como el neurológico o cardiovascular en los pacientes afectados de Covid-19, nos convierte en las siguientes fases de la enfermedad, 1en profesionales esenciales en la recuperación de la autonomía y la calidad de vida ${ }^{(6)}$.

El contexto de aislamiento social por Covid-19 conlleva adquirir estilos de vida no saludables como: Inactividad física, actitud a tener una vida sedentaria, lo que podría incrementar el riesgo de padecer enfermedades no transmisibles a largo plazo y traer consecuencias negativas para la salud frente a la pandemia de la COVID-19.7.

El confinamiento domiciliario decretado por los gobiernos dificulta la participación en los programas de rehabilitación, cuando la duración del confinamiento 
es incierta, se deben encontrar opciones de acceso a necesidades de rehabilitación que podrían conllevar a una disminución funcional no reversible o complicaciones graves ${ }^{(8)}$.

Por último, la terapia física y rehabilitación no solo está presente en la fase de emergencia, así mismo está enfocada en: En la población de riesgo para el Covid-19, personas de edad avanzada y con comorbilidades, durante el periodo de confinamiento inicial, paciente crítico, pacientes hospitalizados, pacientes dados $\mathrm{de}^{(9)}$ alta que padecen Covid 19 , destacando el trabajo del tecnólogo médico en terapia física y rehabilitación con apoyo del equipo multidisciplinario para poder hacer frente a este problema que aqueja tanto a nivel internacional como nacional.

Conflictos de interés: El autor niega conflictos de interés.

Financiamiento: Autofinanciado.

\section{REFERENCIAS BIBLIOGRÁFICAS}

1. Galán-Rodas E, Tarazona-Fernández A PalaciosCeli M. Riesgo y muerte de los médicos a 100 días del estado de emergencia por el COVID-19 en Perú. Acta Médica Peruana.2020; 37(2): 119-121.

2. Colegio de Tecnólogo Médico del Perú. Colegio Tecnólogo Médico del Perú reafirma su compromiso de lucha contra el coranovirus [Internet]. 2020 [citado 5 de Octubre del 2020].

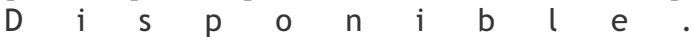
https://ctmperu.org.pe/component/content/ar ticle/8-noticias/27-colegio-tecnologo-medicodel-peru-reafirma-su-compromiso-de-luchacontra-el-coronavirus? Itemid $=101$

3. Colegio de Tecnólogo Médico del Perú. Terapia física y rehabilitación. [Internet]. 2020 [citado 5 de Octubre del 2020]. Disponible https: //ctmperu.org.pe/areas/terapia-fisica-yrehabilitacion.
4. Murieta ES, Supervía M, Covid19 y cronicidad. Una oportunidad de reinventar los servicios de medicina física y rehabilitación, 2020. doi:https://doi.org/10.1016/j.rh.2020.05.002

5. Ríos IDP, Moreno JE, Rodríguez LC, Reyes $M M$,Torres Jl. Fisioterapia respiratoria en la funcionalidad del paciente con covid-19: Respiratory physiotherapy in the functionality of the covid-19 patient. Archivos de Medicina (Manizales).2020; 21(1): 1-16.

6. Rodriguez R, Alcocer MA, Moreno Martínez M, Saenz Espinosa N, La fisioterapia española en tiempos de pandemia del covid19, Fisioterapia. 2020. doi:

https://doi.org/10.1016/j.ft.2020.05.001

7. Avellanet M, Boada-Pladellorens A, Pages-Bolibar E. Rehabilitación En Época De C o n fi $n$ a $m$ i e $n$ t o. 2020 . Rehabilitación.https://doi.org/10.1016/j.rh.202 0.05 .003

8. Bravo-Cucci S, Kosakowski H, Cortés RN, Huamash CS, Asencios JA. La actividad física en el contexto de aislamiento social por COVID-19. GICOS: Revista del Grupo de Investigaciones en Comunidad y Salud. 2020; 5(2): 6-22.

9. Lista-Paz A, González Doniz L, Souto-Camba S. ¿Qué papel desempeña la Fisioterapia en la pandemia mundial por COVID-19?. Fisioterapia.2020; 42(4): 167-169.

\section{Correspondencia}

Santiago Bazán Cristhian

Dirección: Jr Tiahuanaco 1493 Urb. Zárate S.J.L. Lima Perú.

Teléfono: 997602814

Correo: cristhiansantiagob@gmail.com

\section{Revisión de pares}

Recibido: 08/10/2020

Aceptado: 15/12/2020 